\title{
The Level of Use and Effects of Facebook among Young People in Kosovo
}

\author{
MsC. Islam Borinca \\ MsC. Amir Maliqi \\ Department of Psychology, University of Prishtina "Hasan Prishtina", Prishtine, Kosovo \\ Email: Islamborinca@gmail.com, amirshmaliqi@gmail.com
}

Doi:10.5901/jesr.2016.v6n1p198

\begin{abstract}
Facebook as a social factor and with very influential, has a high level of use, especially by teenagers. Consequently, in recent years numerous studies have been conducted, which, among others aim to review and analyze the positive and negative effects stemming from its use. The hypotheses in this study were: Increased use of Facebook has negative effects on academic performance and causes the dependency. While another hypothesis is: One of the main reasons to use Facebook is the tendency of young people to be in the spotlight with their peers. From obtained results it is concluded that, Facebook has negative effects on academic performance and could cause the dependency. In this regard, we have gender differences where, for a nuance girls lead compared with boys. In addition, one of the main reasons of using FB by youths is to be in the spotlight with their peers. Further, reasons why they have chosen to have accounts on FB: Family members and Friends were the primary indicators in this aspect, stressing that their main activity is the conversation through FB, also, they have high expectations to get "likes" from their friends about their posts, statutes or labels, especially during the weekends.
\end{abstract}

Keywords: Facebook, students, academic performance, dependency, spotlight.

\section{Introduzione}

L'adolescenza è una fase della vita nella quale l'individuo acquisisce, le capacita e le conoscenze per le responsabilità della vita da adulto. Le esperienza sociali ed emotivee i cambiamenti del corpo,assumono un grande peso nei i nuovi rapporti con il mondo e se stessi (Kaza, 2006).

L'adolescenza è considerato un periodo di turbolenze, problematiche e tensioni e allo stesso tempo un periodo di grandi opportunità sociali, emozionali, fisiche e cognitive (Reçka, 2005).

Alcuni grandi cambiamenti che accadono nel periodo dell'adolescenza sono i cambiamenti delle emozioni: gli adolescenti possonovariare velocemente la loro disponibilità, possono essereriluttanti, felici, avere paure, variare la propria fiducia e le proprie preoccupazioni riguardo l'identità personale. L'adolescenza è il momento in cuii giovani cominciano ad esplorare e a reclamare la loro identità personale, e in questo periodo di sviluppo, i giovani si impegnano ad un processo di ricerca dove si adattano con i loro coetanei e la società in generale. Relazioni Sociali - durante l'adolescenza le relazioni sociali cominciano a prendere le priorità sulle relazioni con la famiglia, ed influiscono molto circa le loro convinzioni e comportamenti. L'indipendenza e il test dei limiti -gli adolescenti ogni tanto provocano i genitori e gli insegnanti rispetto le regole e i limiti; anche se questo atteggiamento ribelle può sembrare conflittuale ai genitori, in molti casi questo in realtà viene dalla necessità degli adolescenti di avere autonomia e ad essere al centro dell'attenzione. Gli adolescenti possono essere concentrati sui loro bisogni, senza tenere conto come i loro bisogni influisconosu gli altri(Fustrenberg, 2005).

Le pagine dei social networks sono un tipo di tecnologia che sta influendo nella vita privata, in particolare nei giovani, econtemporaneamente danno la possibilità alle persone di collegarsi con diversi individui e attività in tutto il mondo (Vasalou, 2010). Questi social networks con le loro piattaforme possono influire nei giovani dal punto di vista dell'aspetto educativo sia positivamentesia negativamente (Body\&Ellison, 2008).

Unodei social networkpiù famosi nella rete èFacebook, in cui le persone creano i loro profili personali, e dopo si connettono con i profili delle persone che conoscono, sia familiari, amici, colleghi oppure sconosciuti (Special \& Barber,2012)e questo collegamento si manifesta con conversazioni, condivisioni, commenti e mi piace, attività che prende miliardi di click al giorno. Infine vi sono poile foto,che rispecchiano informazioni e interessi personali che possono essere visti o letti dagli altri (Muise, Christofides \& Desmarais, 2009).

Secondo Junco \& Mastrodicasa (2007), la maggior parte degli utenti di Facebook sono giovani e allo stesso tempo 
la principale richiesta di Facebook è la diffusione di vari contenuti, scambio di informazioni eperfezionamento dell'uso con lo scopo del futuro incremento del suo consumo.

Una delle ipotesi principali di questa ricerca èche il frequente uso di Facebook influisce negativamente nella performance scolastica e porta alla dipendenza tra i giovani.

La performance scolastica rispecchia le loro abilità nelle materie scolastiche, la quale è collegata all'assunzione e al tempo che si dedica (Bennett, 2007).

Prensky (2003) ha messo in evidenza la familiarità e il supporto che i giovani, fin da piccoli, acquisiscono riguardo la tecnologia della comunicazione e le informazioni,acquisendo una moltitudine di competenze tecnologiche, sviluppando strategie e diventando anche capaci di manipolare immagini. Oggi i giovani usano Google, You Tube e Facebook mentre svolgono i compiti di scuola a casa però in realtà non sappiamo quanto vengono usate 0 se vengono utilizzate nella maniera giusta (Veen \&Vrakking, 2006).

Pasek (2009)accenna che tante ricerche empiriche parlano di una correlazione negativa tra Facebook e performance scolastica, e i giovani d'altro canto non riescono a generare varie strategiee questo sta influendo negativamente nell'elaborazione dell'informazione.

Gemmill (2006) afferma che il tempo passato dai giovani su Facebook li allontana dalla lororesponsabilitànello studio. In una ricerca dell'Università dell'llinois, i giovani affermano di stare la maggior parte del tempo su Facebook,vedendo questo come un bisogno di notorietà e interazione e non come un indicatore riguardo lo studio(Kennedy, Dalgarno, Gray, Judd, Waycott, Bennett, Maton\& Krause, Bishop, Chang \&Churchward, 2007).

Secondo Lipsman (2008) l'uso esagerato di Facebook influisce nella performance dei giovani dato che essi sono rinchiusi dentro attività e processi extra-scolastici, mentre un'estroversione sociale come l'impegno socialespesso non viene visto come priorità nel processo di studio.

Kisrchner (2009) nel suo studio riguardo la performance e i giovani, ha concluso tali risultati dove i giovani dichiaratisi utenti estremamente attivi di Facebook avevano voti più bassi paragonati agli utenti raramente attivi. Ciò chiarisce che la performance e l'impegno su Facebook hanno una correlazione negativa l'un l'altra a causa dell' impegno dei giovani in due processi simultanei i quali richiedono energie.

II concetto di dipendenza viene definito come un'attività che si caratterizza da esagerazione, tolleranza, astinenza,mancanza, perdita del controllo (Associazione Americana di Psichiatria, 2000). Negli ultimi anni sono aumentate le preoccupazioni riguardoall'uso esagerato dei giovani verso Facebook, i quali stanno diventando sempre più dipendenti e al di fuori della vera vita sociale e non virtuale (Bilton, 2010).

Cassady (2011) nella sua ricerca riguardo a questo tema,ha concluso che come conseguenza dell'accesso troppo facile a Facebook e dell'alto uso da parte dei giovani si stia manifestando una dipendenza, facilitata dalle numerose attività e interazioni che Facebook offre, e ha dimostrato che la maggior parte dei giovani partecipanti alla ricerca non potevanoimmaginare la vita senza Facebook e che tutti esprime vanoun desiderio di rimanere collegati. Infine una delle altre ragioni d'uso la quale porta a dipendenza, è l'uso di Facebook per dimenticarsii problemi personali e familiari, come anche molte persone hanno dichiarato di essere rimproverati da parte dei familiari riguardo l'uso prolungato di Facebook. La dipendenza è considerata come disturbo ed è una nuova diagnosi aggiunta al DSM-V (Associazione Americana di Psichiatria, 2009).

Molte ricerche si sono concentrate sulla questione che i giovanistiano ricevendo tantissime informazioni e chesono estremamente attivi nelle varie condivisioni su Facebook,e da qui nasce la domanda: questa tendenza ha a che fare con il desiderio di essere al centro dell'attenzione dei propri coetanei? (Dimicio, 2007).

Jonison (2008) ha argomentato che i giovani non valutano la propria privacy edè come se essi siano pronti a donare le proprie informazioni per ricompense piccolissime. II ruolo di gestione delle impressioni, la gestione del modo in cui gli altri ti vedono, è anch'esso un fattore importante. Se per esempio una ragazza condivide su Facebook che sta andando in Europa in vacanza, questo potrebbe essere un tentativo per presentare se stessa in un modo particolare, quindi lo scopo di tale condivisione potrebbe essere il presentarsi come una persona colta, non attribuendo alcuna importanza alla condivisione dell'informazione stessa. (Tong, Heide \& Langwell, 2008).

Secondo Peluchette (2008) i giovani hanno la tendenza nel coltivare una certa impressione su gli altri mediante le condivisioni,i tags,le foto, i post o le locazioni con lo scopo di presentarsi come contemporanei, moderni, civilizzati, non ponendo alcuna importanza alle informazioni che essi rivelano.

I fattori ambientali e interpersonalipossono dare alcune spiegazioni riguardo a queste incoerenze. Possiamo aspettarci che i fattori interpersonali influiscano nella vita privata-elementi delle situazioni e delle relazioni tra le persone. Se per caso un caro amico ci fa una domanda personale, possiamo sentire l'inclinazione di rispondergli, perché noi ci teniamo a loro e perchéla condivisione dell'informazione ci rende piùvicini a loro (Kristofides \& Desmarais, 2009). 
I fattori ambientali come le norme e le aspettative di un certo ambiente possono influenzare il comportamento. Nel caso di Facebook, ci sono tante condivisioni che trasmettono alla gente il messaggio che questo sia un ambiente sicuro dove condivideree che la condivisione sia normale. Gli utenti sono bombardati dall'informazione che gli altri hanno condiviso, e tale situazione incoraggia la gente a condividere di più, senza tenere conto dei loro punti di vista nella vita privata (Sheldon, 2008).

\section{Storia di Facebook}

Facebook è stato creato nel 2004 da Mark Zuckberg, Dustin Moskovitz, Andrew McCollume Chris Hughes come una singola pagina per gli studenti di Harward la quale dopo fuestesaanchead altri studenti di tutte le parti del mondo, subendo costanti modifiche per un approccio più facile e comprensibile nei suoicontenuti. (Facebook, 2008).

Nel 2008 Facebook fu scelto tra le 13 pagine web più popolari al mondoe altamente usato dai giovani come fattore socializzante e fondamentale della propria vita(ComScore, 2008). I dati del 2014 parlano di 1.19 miliardi di utenti, 1.11 milioni di nuovi utenti mensili; in media ogni 10 utenti solo 3 sono inattivi; la durata media della connessione è di 20 minuti;il numero medio di amici è di 130 per utente, circa il 39\% fanno condivisioni e pubblicano foto, e ogni 20 minuti condividono un link 0 hanno richieste di amicizia ed inviano messaggi.Facebook è disponibile in 70 lingue diverse (Facebook, 2013).

\section{Gli Effetti di Facebook}

L'uso di Facebook ha i suoi effetti positivi e negativi, ed è diventato uno tra i fattori sociali più importanti nei giovani con un grande uso anche attraversoi telefonini e grazie anche ai dispositivi wi-fi in vari luoghi, il che si identifica con la crescita dell'interazione e delle attività nei giovani ed anche col fatto che i contatti continui offrono delle nuove amicizie anche lontanenonché un costante contatto con i parenti( Gordon \& Juang, 2007).

FB influisce nello sviluppo virtuale dei giovani, il che si descrive dai commenti incoraggianti che risultano positivi, ed anche nello sviluppo della propriaidentità: la scelta della foto di profilo, la visibilità dei mi piace e dei non mi piace, la classifica e la condivisione di quello che si desidera;inoltre FB si vede come compenso sociale nei giovani i quali hanno delle difficoltà verbali, viene visto come una forma di modellamento dei comportamenti e altri fatti che riflettono le scalesociali e la tendenzanel fare le cose al meglio possibile, moderate e inclusive (Hodgkinson, 2008).

FB Però ha anche degli effetti negativi quali: I'isolamento dei giovani e la perdita di tempo nelle attività che gli amici condividono, la famiglia viene messa da parte e si perde l'interesse per la scuola (Westerman, 2008). Alcuni ultimi studi parlano della questione che i giovani stanno diventando narcisisti come conseguenza della loro concentrazione per fare cose interessanti, per comparire belli e si parla anche della questione della gelosia come conseguenza dellacondivisione delle foto, dei link delle persone che amano oppure che hanno amato (Walther, 2008).

L'alto livello di queste condivisioni e lo scambiodell'informazione stanno portando i giovaniverso esperienze negative (Lampune, 2011). Dagli studi effettuati negli USA si è visto che i giovani hanno dichiarato che hanno avuto delle esperienze negative come: diffamazione sulla loro vita privata, condivisione delle foto intime che normalmente non si mostrerebbero ad amici oppure ai familiari, ma anche messaggi minacciosi o provocanti, falsi profili così come la perdita di tempo e mancanza per altre attività sia scolastiche oppure familiari (Hancock, 2011).

Molti giovani sono testimoni di casi quando i loro amici oppure colleghi pubblicano dei post riguardo ai suicidi e richiedono anchedelle opinioni oppure votazioni se devono compiere l'atto a seguito delle disgrazie che hanno subito (Muise, 2009).

Secondo Binder (2009) come risultato dell'abuso su FB, in molti casi si è avuto bisognodell'intervento della polizia, della famiglia, e della comunità, a causa delle minacce, delle pubblicazioni sul suicidio oppure dell'abuso dei profili, e ciò normalmente serve come una brutta esperienza per i giovani.

Vale la pena dire che in alcuni paesi Facebookè stato censurato e bloccato, l'Australia è uno dei paesi che ha messo le censure per quanto riguarda l'iscrizione, richiede la conferma per l'età di 18 anni, invece in Bangladesh FB viene monitorato dal governo in collaborazione con l'agenziadi intelligence; in Cina FBèstato bloccato a causa dell'utilizzo da parte degli attivisti dell'indipendenza di Xinjagn mentre in alcune zone ne era stata bloccata l'iscrizione Vi sono anche dati di censura e di blocco in alcuni paesi come: Corea del Nord, Cuba,Tagikistan, Egitto, Siria, Iran e anche per II Regno Unito, il quale ha messo la censura in riferimento al matrimonio del principe William e CatherineMiddleton, la qualeè stata travoltada diversi commenti offensivi, seguita da proteste dei diversi gruppi e interventi severi della polizia (Bernal, 2011). 
L'oggetto dello studio: Il livello di utilizzo e gli effetti di Facebook nei giovani di età compresa tra i 14-18 anni nella città di Kamenica.

Lo scopo di questo studio: relazionedegli eventuali legami tra l'utilizzo di Facebook e i suoi effetti nei giovani di età compresa tra 14 - 18 anni nella città di Kamenica.

In concreto:

1. A che livello è l'impatto di Facebook nei giovani.

2. Evidenziare gli effetti positivi e negativi di Facebook nei giovani

3. Conoscere i diversi motivi dell'utilizzo di Facebook nei giovani

Ipotesi

In questo studio ci sono due ipotesi, dove una è primaria invece l'altra si presenta come secondaria. L'ipotesi primaria oppure principale di questo studio è: I/ grande utilizzo di Facebook influisce negativamente nella performance dell'apprendimento e nella dipendenza. Invece l'altra ipotesi è: Uno dei principali motivi dell'utilizzo di FB è la tendenza dei giovani nell'essere al centro dell'attenzione nei confronti dei loro coetanei.

\section{Metodologia}

\subsection{Metodo di ricerca}

Come metodo di ricerca è stato usato il metodo quantitativo. II metodo quantitativo serve per misurare le caratteristiche umane, la modellizzazione statistica, la matematica dei processi psicologici e la realizzazione degli studi di ricerca e l'analisi dei dati psicologici (Newman, 1998). II motivo per cui questa metodologia è stata utilizzata perché è più pratico e di facile accesso soprattutto, per i giovani!

\subsection{I partecipanti}

II numero generale dei partecipanti in questo studio è di 120 alunni.

Questo numero è stato scelto dalla IX, X, XI e XII classe da due scuole che si trovano nella città di Kamenicë Kosovo - (presso la scuola elementare di Fan S. Noli e presso la scuola media superiore Ismail Qemali)

La scelta del campione dei partecipanti è stata fatta in modo casuale per ambedue i generi in collaborazione con $\mathrm{i}$ rappresentanti scolastici e con il permesso del provveditorato agli studi della città.

\subsection{Lo strumento di Misura}

Lo strumento di misura usato in questo studio è il questionario.

Il questionario è una procedura del recupero e di elaborazione dei dati di una specifica tematica della psicologia, che si estende ai membri di una comunità o di uno specifico gruppo sociale. II questionario è composto da domande che vengono rivolte ai soggetti riguardo i dati personali e opinioni, sulla loro posizione e sulle tipologie del loro comportamento legato al problema socio-psicologico che viene esaminato (Nushi, 1999).

II questionario che è stato usato in questa ricerca contiene 21 domande che ci forniranno delle informazioni riguardo l'applicazione, gli effetti, l'influenza di FB nel processo didattico, nella dipendenza e nel ragionamento o lo scopo del suo uso nei giovani.

Il questionario è stato formulato dagli autori di questa ricerca, mentre alcune domande riguardo alla dipendenza sono state recuperate dal questionario dell'autore Bernard Cassady, che nel 2011 aveva esaminato la dipendenza dall'uso di Facebook.

\subsection{II modello sperimentale}

L'elaborazione dei dati è stata realizzata tramite PSPP.

Sulla base dei calcoli effettuati con il programma PSPP di ogni risposta data, si vede chiaramente la conferma delle nostre ipotesi e dopo averli comparati, riusciamo a notare le differenze in base al sesso, l'età, usando il coefficiente alfa, la presentazione delle tabelle etc. 


\subsection{La procedura}

Dopo la selezione dei partecipanti, è stato illustrato lo scopo della ricerca e in concreto riguardo al livello di uso e gli effetti di Facebook nei giovani. È stato riferito che il questionario era in forma anonima e venivae richiesta sincerità nelle risposte. II tempo adisposizione è stato all'incirca di 10-15 minuti.

\section{I Risultati}

I partecipanti in questa ricerca erano 120, dove 55 erano mashi e 65 femmine, con un'età media dai 14-18 anni. In particolare, i partecipanti di 14 anni erano in 19, 10 femmine e 9 maschi, i partecipanti dell'età di 15 anni erano in 23, 12 femmine e 11 maschi, i partecipanti di 16 anni erano in 27, 16 femmine e 11 maschi, i partecipanti di 17 anni erano in 28, 15 femmine e 13 maschi e i partecipanti di 18 anni erano in 23, 12 femmine e 11 maschi.

\subsection{Sesso}

\begin{tabular}{lccccc}
\hline Value label & Value & Frequency & Percent & Valid Percent & Cum Percent \\
\hline Maschi & 1 & 55 & 45.83 & 45.83 & 45.83 \\
Femmine & 2 & 65 & 54.17 & 54.17 & 100.00 \\
Totale & 120 & 120.0 & 120.0 & & \\
\hline
\end{tabular}

\subsection{Età}

\begin{tabular}{cclccc}
\hline $\begin{array}{l}\text { Value Label } \\
\text { Percent }\end{array}$ & Value & Frequency & Percent & Valid Percent & Cum \\
\hline 14 & 1 & 19 & 15.83 & 15.83 & 15.83 \\
15 & 2 & 23 & 19.17 & 19.17 & 35.00 \\
16 & 3 & 27 & 22.50 & 22.50 & 57.50 \\
17 & 4 & 28 & 23.33 & 23.33 & 80.83 \\
18 & 5 & 23 & 19.17 & 19.17 & 100.00 \\
Totale & 120 & 100.0 & 100.0 & & \\
\hline
\end{tabular}

\subsection{Classe}

\begin{tabular}{lccccr}
\hline $\begin{array}{l}\text { Value Label } \\
\text { Percent }\end{array}$ & Value & Frequency & Percent & Valid Percent & Cum \\
\hline K1.IX & 1 & 18 & 15.00 & 15.00 & 15.00 \\
K1.X & 2 & 23 & 19.17 & 19.17 & 34.17 \\
K1.XI & 3 & 45 & 37.50 & 37.50 & 71.76 \\
K1.XII & 4 & 34 & 28.33 & 28.33 & 100.00 \\
Totale & 120 & 100.0 & 100.0 & & \\
\hline
\end{tabular}

\subsection{Cronbach Alpha}

\begin{tabular}{l}
\hline Cronbach Alpha $\quad \mathrm{N}$ of \\
Items \\
\hline
\end{tabular}

.09

21

Il coefficiente alfa è risultato essere .09 e di conseguenza il questionario viene considerato con un alto livello di affidabilità (Conbrach,1951). 


\subsection{T-TEST}

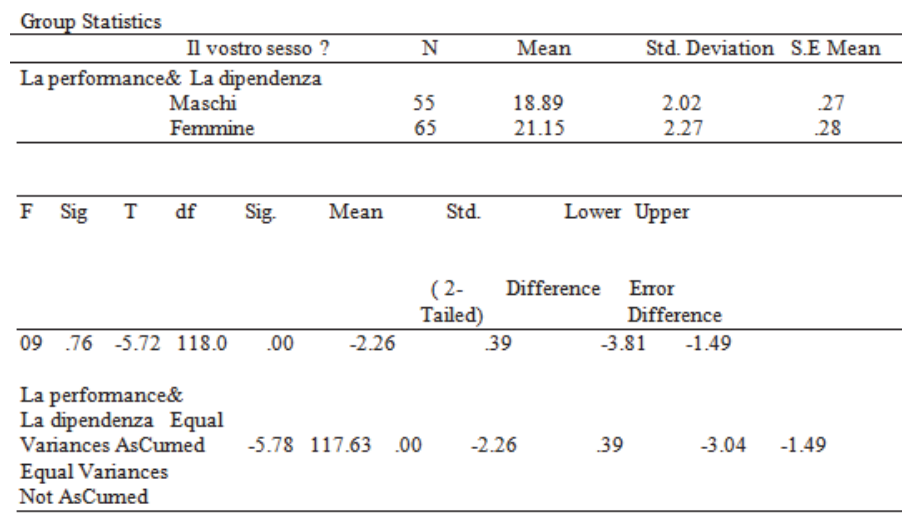

Nell'atteggiamento dei maschi abbiamo notato che è risultato che $X=18.89$ e $D S=2.02$, invece le femmine $X=21.15$ e $D S$ 2.27 e il T-Test è (117.63)- 5.78 e $\mathrm{P}=.00$, quindi abbiamo distinzioni significanti e come risultato del DS è evidente che I'uso frequente di Facebook influisce negativamente nella performance didattica e il livello di dipendenza trova conferma nella nostra ricerca.

\subsection{CHISQ}

\begin{tabular}{|c|c|c|c|c|c|c|}
\hline \multicolumn{7}{|l|}{ Cummary } \\
\hline \multicolumn{6}{|l|}{ Cases } & \multirow[b]{2}{*}{ Total } \\
\hline \multirow[b]{3}{*}{ Il vostro sesso? } & \multirow[b]{3}{*}{ *attenzione } & \multicolumn{2}{|r|}{ Valid } & \multicolumn{2}{|c|}{ Missing } & \\
\hline & & \multicolumn{2}{|c|}{ T Valla } & $\mathrm{N}$ & Percent & N Percent \\
\hline & & 120 & $100.0 \%$ & 0 & $0.0 \%$ & $120 \quad 100.0 \%$ \\
\hline \multicolumn{7}{|c|}{ Il sesso *attenzione [count, row $\%$, column $\%$, total $\%$ ]. } \\
\hline \multicolumn{7}{|c|}{ L'attenzione } \\
\hline Il vostro sesso? & 1 & & & 2 & & Totali \\
\hline \multirow{4}{*}{ Maschi } & 33.00 & & & 22.00 & & 55.00 \\
\hline & $60.00 \%$ & & & $40.00 \%$ & & $100.00 \%$ \\
\hline & $39.76 \%$ & & & $59.46 \%$ & & $45.83 \%$ \\
\hline & $27.50 \%$ & & & $18.33 \%$ & & $45.83 \%$ \\
\hline \multirow[t]{4}{*}{ Femmine } & 50.00 & & & 15.00 & & 65.00 \\
\hline & $76.92 \%$ & & & $23.08 \%$ & & $100.00 \%$ \\
\hline & $60.24 \%$ & & & $40.54 \%$ & & $54.17 \%$ \\
\hline & $41.67 \%$ & & & $12.50 \%$ & & $54.17 \%$ \\
\hline \multirow[t]{4}{*}{ Totale } & 83.00 & & & 37.00 & & 120.00 \\
\hline & $69.17 \%$ & & & $30.83 \%$ & & $100.00 \%$ \\
\hline & $100.00 \%$ & & & $100.00 \%$ & & $100.00 \%$ \\
\hline & $69.17 \%$ & & & $30.83 \%$ & & $100.00 \%$ \\
\hline
\end{tabular}

\subsection{Chi-square tests.}

\begin{tabular}{lccc}
\hline Statistic & Value & $d f$ & Asymp. Sig. (2-tailed) \\
\hline Pearson Chi-Square & 4.00 & 1 & .05 \\
Likelihood Ratio & 4.00 & 1 & .05 \\
Continuity & 3.25 & 1 & .07 \\
Correction & 3.97 & 1 & .05 \\
Linear-by- & 120 & & \\
Linear Association & & & \\
N of Valid Cases & & & \\
\hline
\end{tabular}


Dal comportamento dei maschi è risultato che $X=39.76 \%$ e dalle femmine $X=60.24 \%$, invece $\mathrm{CHIS}\left(X^{2}\right)=4.00$ e $P=.05$, quindi in questo caso abbiamo distinzioni significative che confermano la seconda ipotesi: Uno dei molteplici motivi dell'uso di Facebook è la tendenza dei giovani ad essere al centro dell'attenzione rispetto ai loro coetanei, e questa ipotesi trova conferma nella nostra ricerca.

Alla domanda: Qual'è stato il livello dei risultati scolastici nella prima metà dell'anno, 60 5.00\% degli studenti hanno risposto di avere avuto poco successo, 4 partecipanti o il 3.33\% ha risposto di avere avuto successo sufficiente, invece 34 o il 28.33\% avevano avuto un buon livello di successo, 33 o il 27.50\% ha avuto un livello molto buono di successi e invece 43 o il 35.83\% hanno avuto un livello eccellente di successi.

Alla domanda: Quanto tempo passate a studiare, 25 di loro o il 20.83\% dei partecipanti ha dichiarato di studiare meno di 30 minuti, 30 partecipanti o il $25.00 \%$ ha dichiarato tra 30 e 60 min, 45 partecipanti o il $37.50 \%$ ha dichiarato 1-2 ore e 20 partecipanti o il $16.67 \%$ ha dichiarato di studiare piu di 2 ore.

Alla domanda: Riguardo al possesso dell'account di Facebook. Tutte le persone hanno dichiarato di averne uno, quindi tutti i 120 o il 100\%.

Alla domanda: Da quanto tempo avevano un account Facebook. 13 partecipanti o il 10.83\% ha risposto di aver aderito meno di un anno fa, 23 persone o il 19.71\% hanno aderito 1-2 anni fa, 48 partecipanti o il 40.00\% erano attivi da più di 4 anni.

Alla domanda: Quanto tempo passate su Facebook, 27 partecipanti o il 22.50\% ha dichiarato di passare meno di un ora, 26 o il $21.67 \%$ 1-2 ore, 37 o il $30.83 \%$ vi passa $2-3$ ore e 30 o il $25 \%$ lo utilizza più di 4 ore.

Alla domanda: Riguardo al motivo dell'apertura di un account FB, 44 partecipanti hanno dichiarato a causa degli amici, 7 partecipanti o il 5.83\% a causa dei familiari e 60 o il 50\% perchè gli piace FB.

Alla domanda: Riguardo ai posti preferiti per accedere a FB, 93 partecipanti o il $77.50 \%$ ha dichiarato da casa, 3 partecipanti o il 2.50\% da scuola, 22 partecipanti o il 18.33\% da un bar, 2 partecipanti o il 1.67\% da altri posti.

Alla domanda: Se i familiari se la prendono con voi per il tempo passato su FB, 20 partecipanti o il 16.67\% dei partecipanti ha dichiarato di non essere mai stato rimproverato, 60 partecipanti o il 50.00\% ogni tanto e 40 partecipanti 0 il 33.33\% molto frequentemente.

Alla domanda: Se vi è mai successo che la lezione non fosse interessante e avete passato il tempo su FB, 36 partecipanti o il $30.00 \%$ ha dichiarato mai, 58 partecipanti o il $48.33 \%$ ogni tanto e 26 partecipanti o il $21.67 \%$ molto spesso.

Alla domanda: Vi capita che passate tanto tempo senza accedere a FB, 24 partecipanti 0 il $20.00 \%$ dichiara di si, invece 96 partecipanti o il $80 \%$ ha dichiarato di no.

Alla domanda: Se vi è capitato di finire velocemente le vostre attività per poter accedere a FB, 27 partecipanti 0 il $22.50 \%$ ha risposto mai, 65 partecipanti o il $54.17 \%$ ogni tanto e 28 partecipanti o il $23.33 \%$ ha risposto molto spesso.

Alla domanda: Se la vita vi sembra noiosa o monotona senza FB, 76 partecipanti o il $63.33 \%$ ha risposto si e 44 partecipanti o il $36.67 \%$ ha risposto no.

Alla domanda: Secondo voi, chi non ha FB viene considerato non attuale, 59 partecipanti o il $49.17 \%$ ha risposto SI e 61 o il 50.83\% ha risposto NO.

Alla domanda: Cercate di essere attrattivi durante le conversazioni, durante la pubblicazione 0 condivisione dei post, 79 partecipanti o il $65.83 \%$ ha risposto si e invece 41 partecipanti o il $34.17 \%$ ha risposto no.

Alla domanda: Se vi è mai capitato di avere esperienze negative su FB, 32 partecipanti 0 il $26.67 \%$ ha risposto di si, a causa dei furti di account FB, 23 partecipanti o il 19.17\% per abuso di uso delle foto, 63 partecipanti o il $52.50 \%$ a causa di messaggi minacciosi e 2 partecipanti o il 1.67\% per altri motivi.

Alla domanda: Quante volte al giorno controllate FB, 35 partecipanti o il 29.17\% ha dichiarato 0-30 minuti, 27 partecipanti o il 22.50\% 30-60 minuti, 58 partecipanti o il 48.33\% 1-2 ore.

Alla domanda "Vi e mai successo di entrare su Facebooksolo per 5 minutie di rimanerci più a lungo"hanno risposto "si" in 109 (90.83\% dei partecipanti) mentre hanno risposto "no" in 11 (9.17\% dei partecipanti).

Alla domanda "In quale giorno della settimana pensate di ricevere più mi piace o commenti nelle vostre pubblicazioni dei post" in 102 (85.00\% dei soggetti)hanno risposto in fine settimana, mentre in 18 (15.00\% dei soggetti) hanno risposto durante le giornate lavorative.

Alla domanda "Quando distribuisco foto, status, tags, mi aspetto tanti mi piace dagli amici"hanno risposto "si" in 83 (69.17\%) e "no" in 64 (30.83\% dei partecipanti).

Alla domanda "vi sentite a vostro agio nei posti dove non c'è il Wi-Fi" hanno risposto "si" in 56 (46.57\% dei partecipanti)mentre "no" in 64 (53.33 \% dei partecipanti).

Alla domanda "Che attività svolgete più spesso su Facebook" 17 ovvero il 14.17\% ha risposto "condivisione", 29 
ovvero il $24.17 \%$ ha risposto "mi piace e commenti", 67 ovvero il $55.83 \%$ ha risposto "chat" e 7 ovvero il $5.83 \%$ ha risposto "altre attività"

\section{Discussione}

Dallo studio si evidenzia che tutti i partecipanti usano Facebook e nonostante la maggior parte di essi riferisca risultati ottimi per il semestre,le analisi statistiche dimostrano che Facebook influisce negativamente nella performance scolasticae crea dipendenza. Allo stesso tempo si notano minime differenze riguardo il genere, anche se le ragazze adolescenti sono in più dei ragazzi riguardo questo aspetto.Le analisi statistiche mostrano che una delle ragioni per cui si usaFacebookè la tendenza di voler essere al centro dell' attenzionenei confronti dei propri coetanei.

II tempo che si passa studiando è30-60 minuti al giorno, mentre il tempo dedicato a Facebook si aggira sulle 2-3 ore con una controllabilità di 1-2 ore.

Un'alta percentuale di giovani dichiara di essere rimproverata dai familiari per il tempo speso su Facebook, ed è stato scoperto che a scuola, quando la materia non risulta essere attrattiva, un'alta percentuale di loro si connette a Facebook.

L'importanza di Facebook per i giovani viene dimostrata dal fatto che un'altissima percentuale di loro non riesce a stare disconnesso da Facebook e che la vita senza di esso gli sembra monotona e noiosa, anzi preferiscono finire velocemente le altre attività solo per poi poter entrare su Facebook. Allo stesso tempo, un'alta percentuale di giovani entra su Facebook con l'intenzione di restarci solo per 5 minuti ma poi finisce a rimanerci per ore intere.

Per quanto riguarda le ragioni per cui ci si connette a Facebook, gli amici e i familiari erano di principale importanza, accennando che la principale attività su Facebook sia il chatting, mentre un'alta percentuale si aspetta "mi piace"dagli amici riguardo i post, gli status oppure i tag, principalmente durante il fine settimana.

I posti preferiti per la connessione a Facebook sono le case in un'alta percentuale, seguite dai bar,ma vi sono anche dichiarazioni di connessione negli ambienti scolastici. Ė stato anche affermato di non sentirsi a proprio agio in posti senza wi-fi.

Vale accennare che c'è una percentuale uguale tra coloro che dichiarano che chi non possiede Facebook sia non attuale o meno, mentre c'è un'altissima percentuale di coloro che dichiarano di cercare di essere attrattivi su Facebook durante le loro interazioni, siano queste chat, mi piace o commenti.

II presente studio rispecchia anche le esperienze negative tra i giovani, causate dall'uso eccessivo. Tra queste i messaggi di minaccia e la violazione degli account tenevano i primi posti.

I risultati del presente studio sono simili a quelli di tanti studi internazionali riguardo Facebook: Lo studio fatto nel 2010 nell'Università dell'Ohio in USA, che riguardava l'influenza di Facebook nella performance scolastica ha portato alla conclusione che i giovani prestano più tempo su Facebook che alla preparazione scolastica, che, i giovani si preoccupano delle attività e delle interazioni su Facebook, perdendo la flessibilità verso gli obblighi scolastici, e sottolinea l'inizio dell'uso di Facebook all'interno degli ambienti scolastici (Kirschner \& Karpinski,2010).

Allo stesso tempo, un altro studio effettuato nel 2010 dall'Università dell'Utah in USA, riguardo Facebook e la performance scolastica, ha concluso che essa si sta mettendo in pericolo dall'uso eccessivo di Facebook da parte dei giovani alludendo ad un legame emozionale-virtuale che si sta creando verso esso; inoltre gli altri processi, come la performance scolastica e addirittura le interazioni familiari sono in caduta (Bjerregaard, 2010).

Lo studio effettuato nel 2011 da Bryan Casady, lettore presso l'Università dell'Indiana negli USA riguardante Facebook e la dipendenza da esso, ha concluso che i giovani stanno creando dipendenza da esso. Questo viene dimostrato dal fatto che i giovani vengono sempre rimproverati dai parenti per il tempo speso su Facebook. Essi addirittura entrano per restare solo 5 minuti, però finiscono a passarci ore intere e non si sentono a proprio agio in posti senza wi-fi. Simili risultati sono stati ottenuti anche dal presente studio in questo aspetto (Casady, 2011).

Un altro studio effettuato nel 2009 dall'Università del Guelph in Canada, riguardo la privacy e i post pubblicati dai giovani su Facebook, ha concluso che i post, i commenti, i mi piace, si fanno con l'intento di attrarre l'attenzione dei coetanei e che i giovani stanno vivendo esperienze negative dall' uso eccessivo come violazione degli account, messaggi offensivi, minacce, messaggi seduttivi e, ultimamente anche dichiarazioni di suicidio da parte di giovani, i quali stanno causando scosse psichiche nei loro coetanei (Christofides, Muise \& Desmarais,2009).

Lo studio effettuato nel 2013 dall'Università di Birmingham in Inghilterra, riguardo agli usi di Facebook, i tag, i posttra i giovani, ha concluso che Facebook viene enormemente usato dai giovani e dichiarano che in tal modo si sentono allo stesso passo con il mondo ed in contatto con il mondo e la società, mentre le loro attività preferite su Facebook sono la pubblicazione di foto, conclusione differente dal nostro studio, dal quale è risultato chele chat fossero 
le attività preferite. (Houghton, Jonison, Caldwell, 2013).

Infine, vale la pena notare che i genitori possono essere in grado di ridurre gli effetti dei mass media (e.g, TV), per i loro figli, a discutere il loro comportamento e gli atteggiamenti (Borinca, 2015), ma oggi l'uso di siti di networking è più privato a causa del dispositivo elettronico (cellulare, iPhone, iPad, iPod) diverso, sulla base di questo, per i genitori è molto difficile a volte per capire che cosa i loro bambini stanno facendo e per controllarla!

\section{I Limiti e le Raccomandazioni}

I limiti dello studio riguardano il campione che è stato selezionato solo dalle scuole che si trovano dentro la città di Kamenicë,omettendo i villaggi circostanti, però i risultati possono essere generalizzati anche per i giovani dei villaggi siccomela maggior parte di loro va a scuola in città e ci passa la maggior parte del suo tempo, e anche i sistemi di connessione a internet sono presenti anche nei villaggi ormai.

\subsection{Le raccomandazioni}

Raccomandiamo lo svolgimento di altri studi eseguiti da esperti riguardo alla questione, con un maggior supporto istituzionaledal qualerisulterebbero ulteriori raccomandazioni.

Raccomandiamo studi a livello nazionale. Che i giovani vengano messi a conoscenza degli aspetti positivi e negativi, dagli insegnanti e dalla presenza e supporto di psicologi nelle scuole.

Raccomandiamo lezioni per l'incremento della consapevolezza da parte degli psicologi delle scuole o degli insegnanti riguardo all'uso effettivo di Facebook. I parenti dovrebbero essere più vigilanti rispetto al tempo trascorso dai loro figlie le attività che loro svolgono su Facebook.

\section{References}

American Psychiatric Association (2000).Diagnostic and statistical manual of mental disorders: DSM-IV-TR.4th ed. Washington, DC: American Psychiatric Association

American Psychiatric Association (2009).Diagnostic and statistical manual of mental disorders: DSM-V-TR.1th ed. Washington, DC: American Psychiatric Association

Belstat Census (2005). Enquêtebelgesurl'emploi du temps.Scaricato il 22 Gennaio, 2014 dal sito http://www.timeuse.be/tostat/table.php?lang=fr\&extrapol=1\&pool=1\&ind=1\&ind=2\&ind=3\&act0=4\&cxt=act1Bilton.

Bernal, P. (2011). Web Spies, Index on Censorship: For Free Expression, vol. 40, no. 2 , p. 112.

Binder, J., Howes, A., \& Sutcliffe, A. (2009). The Problem of Conflicting Social Spheres: Effects of Network Structure on Experienced Tension in Social Network Sites. Articolopresentatoalla CHI2009, Boston, MA, USA.

Bjerregaard M. (2010).Facebook's effects on subtle emotion decoding, academic performance and identity protection. Master Student Thesis Southern Utah University 60,1-30

Borinca, I. (2015). The Impact of Television on Sexual Attitudes and Behaviors among Teenagers, Ages 15 - 17 Years Old, in the City of Klina, Kosovo. Psychology, 6, 700-707. http://dx.doi.org/10.4236/psych.2015.66068

Boyd, D.M. (2007). Why youth (heart) social network sites: The role of networked publics in teenage social life, In MacArthur Foundation Series on Digital Learning - Youth, Identity, and Digital Media Volume, edited by D. Buckingham, 119-142. Cambridge, MA: MIT Press.

Brandtzæg, P. B., Lüders, M. \&Skjetne, J. H. (2010).Too Many Facebook "Friends"? Content Sharing and Sociability Versus the Need for Privacy in Social Network SiteInternational Journal of Human-Computer Interaction, 26(11-12), 1006-1030.

Cassady,B.(2011) .The Facebook Addiction Test: A new tool to Study FacebookUsage, 1(1),1-13

ComScore.(2008). Comscore top 50 properties (U.S.) http://www.comscore.com/press/data/top_50_web_properties.asp, consultato il 20 Gennaio 2014.

Conbrach ,L.J.(1951).Coefficient alpha and internal structure of tests. Psychometrika, 16(3),297-334.

Christofides, E., Muise, A., \&Desmarais, S. (2009). Information disclosure and control on Facebook: Are they two sides of the same coin or two different processes? CyberPsychology\& Behavior, 12(3), 341-345.

DiMicco, J. M., \&Millen, D. R. (2007). Identity Management: Multiple Presentations of Self In Facebook.Articolopresentatoalla Proceedings of the 2007 International Association for Computing Machinery conference on Supporting group Work, Sanibel Island, Florida, USA.

Ellison, N., Steinfield, C., \& Lampe, C. (2007). The benefits of Facebook " Friends": Social Capital andCollege Student's Use of Online Social Network Sites. Journal of Computer-MediatedCommunication, 12(3), 1143-1168.

Facebook (2008). Statistics.http://www.facebook.com/press/info.php?statistics , consultato il 21 Gennaio 2014.

Facebook (2014).Statistics. http://www.facebook.com/press/info.php?statistics, consultato il 28 Gennaio 2014

Furstenberg, F., Rumbaut, R. G., \&Settersten, R. A. (2005). On the frontier of adulthood: An introduction. In R. A. Settersten, F. 
Furstenberg, \& R. G. Rumbaut (Eds.), On the frontier of adulthood: Theory, research, and public policy (pp. 3-28). Chicago: University of Chicago Press.

Gemmill, E., \& Peterson, M. (2006). Technology use among college students: Implications for student affairs professionals. NASPA Journal, 43, 280-300.

Gonzales, A. L., \& Hancock, J. (2011). Mirror, Mirror on my Facebook Wall: Effects of Exposure to Facebook on Self-Esteem. CyberPsychology\& Behavior, 14(1/2), 79-83.

Gordon, C. F., Juang, L. P., \& Syed, M. (2007). Internet use and well-being among college students: beyond frequency of use. Journal of College Student Development, 48(6), 674-688.

Hodgkinson, T. (2008).With friends like these...The Guardian. http://www.guardian.co.uk/technology/2008/jan/14/facebook , consultato il 14 Marzo, 2014,

Houghton,D.,Jonsion,A.,Caldwell,N.(2013). Tagger's delight? Disclosure and liking in Facebook: The effects of sharing photography amongst multiple known social circles 6,1-45

Joinson, A. N. (2008). 'Looking at', 'looking up' or 'keeping up with' people? Motives and uses of Facebook. Paper presented at the CHI 2008 - Online Social Networks, Florence, Italy,34,(1),4855.

Junco, R. \&Mastrodicasa, J. (2007).Connecting to the net.generation: What higher education professionals need to know about today's students. NASPA.

Karpinski, A. C. \& Duberstein, A. ( 2009 ). A description of Facebook use and academic performance among undergraduate and graduate students. Poster presented at the meeting of the American Educational Research Association, San Diego, CA.

Kennedy, G., Dalgarno, B., Gray, K., Judd, T., Waycott, J., Bennett, S., Maton, K., Krause, K.-L., Bishop, A., Chang, R., \&Churchward, A. (2007). The netgeneration are not big users of Web 2.0 technologies: Preliminary findings. In R. J. Atkinson, C. McBeath, S. $\mathrm{K}$.

A. Soong,.\& C. Cheers (Eds.), ICT: Providing choices for learners and learning.Proceedings of ASCILITE 2007 Conference. Centre for Educational Development, Nanyang Technological University, Singapore. [Online], http://www.ascilite.org.au/conferences/singapore07/procs/kennedy.pdf consultato il15 Marzo, 2014

Kaza,N. (2006) Adoleshenca Stina e Shpërthimev. Shtypshkronja "Migeeralb". Tiranë.

Kirschner, P. A., \&Karpinski, A. C. (2010) Facebook and academic performance. Computers in Human Behavior, 26, $1237-1245$.

Kolek, E. A., \& Saunders, D. (2008). Online disclosure: An empirical examination of undergraduate Facebook profiles. NASPA Journal, 45(1), $1-25$

Lampinen, A., Lehtinen, V., Lehmuskallio, A., \&Tamminen, S. (2011). Were in it Together:Interpe rsonal Management of Disclosure in Social Network Services.Articolo presentato alla CHI 2011, Vancouver, Canada.

Lipsman, A. (2007a, June). Facebook sees flood of new traffic from teenagers and adults.

Muise, A., Christofides, E., \&Desmarais, S. (2009). More information than you ever wanted to know - Does Facebook bring out the green-eyed monster of jealousy? CyberPsychology\& Behavior, 12(4), 441-444.

Newman, I. \& Benz, C.R. (1998). Qualitative-Quantitative Research Methodology: Exploring the Interactive Continuum. Carbondale, IL: Southern Illinois University Press.

Nushi, P (1999). Psikologji e përgjithshme I, Njeriu dhe personaliteti i tij in Psikologji, Universitetit i Prishtines,Prishtinë.

Pasek, J., More, E., \&Hargittai, E. (2009, May). Facebook and academic performance: Reconciling a media sensation with data.First Monday, 14(5).

Reçka,L (2005).Psikologjia e zhvillimit. Casa Editrice "Libri Universitar".Tiranë.

Peluchette, J., \& Karl, K. (2008) Social networking profiles: An examination of student attitudes regarding use and appropriateness of content. CyberPsychology\& Behavior, 11(1), 95-7.

Prensky, M. (2003).Digital game-based learning. ACM Computers in Entertainment, 1(1), 1 - 4.

Special, W. P., \& Li-Barber, K. T. (2012). Self-disclosure and student satisfaction with Facebook.Computers in Human Behavior, 28(2), 624-630

Sheldon, P. (2008). Student favorite: Facebook and motives for its use. Southwestern Mass Communication Journal, 23,(2) 39-55.

Tom Tong, S., Van Der Heide, B., \&Langëell, L. (2008).Too much of a good thing?

The relationship between number of friends and interpersonal impressions on Facebook.Journal of Computer-Mediated Communication, $13,(1) 531-49$.

Vasalou, A., Joinson, A., \& Courvoisier, D. (2010). Cultural differences, experience with social network sand the nature of "true commitment" in Facebook. International Journal of Human-ComputerStudies, 68(10), 719-728.

Veen, W., \&Vrakking, B. (2006). Homo Zappiens: Growing up in a digital age. London, Network Continuum Education.

Walther, J. B., Van Der Heide, B., Kim, S.-Y., Westerman, D., \& Tong, S. T. (2008). The role of friends' appearance and behavior on evaluations of individuals on Facebook: Are we known by the company we keep? Human CommunicationResearch, 34(1), 2849. 